\title{
New Developments in RISE Microscopy: Correlative Raman and SEM Imaging
}

\author{
Ute Schmidt $^{1}$, Philippe Ayasse ${ }^{1}$ and Olaf Hollricher ${ }^{1}$ \\ 1. WITec GmbH, Ulm, Germany (www.witec.de)
}

Instruments that incorporate the strengths of several measurement techniques facilitate a more comprehensive sample characterization. The combination of a fully automated confocal Raman Imaging microscope and a stand-alone Scanning Electron microscope (SEM), known as a RISE microscope, has demonstrated an ability to provide insight and detail beyond that available with the constituent methods individually.

RISE microscopy can now be added to fully equipped SEMs from Tescan or Zeiss, with both microscopes allowing a precise transfer of the sample inside the vacuum chamber. The Raman imaging component in RISE microscopes is designed for ease of use without compromising confocality, lateral and spectral resolution, or acquisition speed for large spectral data sets. Laser wavelength selection with a mouse click and absolute laser power determination in $0.1 \mathrm{~mW}$ increments are accessible through an intuitive user interface. The integrated RISE software carries out the required parameter adjustments and instrument alignments, including grating selection in wavelength-optimized spectrographs. The acquired results are then correlated and the Raman and SEM images overlaid to form RISE images. It is therefore possible to visualize the chemical nature of the sample surface structures investigated with nanometer resolution using SEM.

In contrast to conventional systems in which individual Raman spectra are collected from areas several micrometers across, RISE can produce diffraction-limited confocal Raman images from the same submicron areas investigated by SEM. It can also generate 3D-Raman images and depth profiles to describe the distribution of the molecular compounds within a sample volume.

In Fig. 1 a correlative Raman-SEM-EDX measurement from a geological application is presented. This example shows analysis of the mineral phases of a rock section from a drill core [1]. The SEM image (Fig. 1a) was acquired with a field emission SEM from Tescan. An EDX (Fig 1b) and a Raman image were also acquired from the same sample position. Fig. 2 shows an example from battery research highlighting the RISE integration with a Gemini SEM from Zeiss [2].

The aim of this presentation is to present the versatility of RISE microscopy and to highlight achievements from the past year.

\section{References:}

[1] J. Wahlgren, P. Curtis, C. A. Triumf, H. Drake, E. L. Tulborg „, Geological description of rock domains and deformation zones in the simpevarp and Laxemar subarea - Version 1.2" No. R-05-69 (Stockholm 2006)

[2] S. Freitag, C. Berger, C. Weisenberger, A. Kopp, U. Schmidt, P. Ayasse Zeiss Application Note, October 2015. 

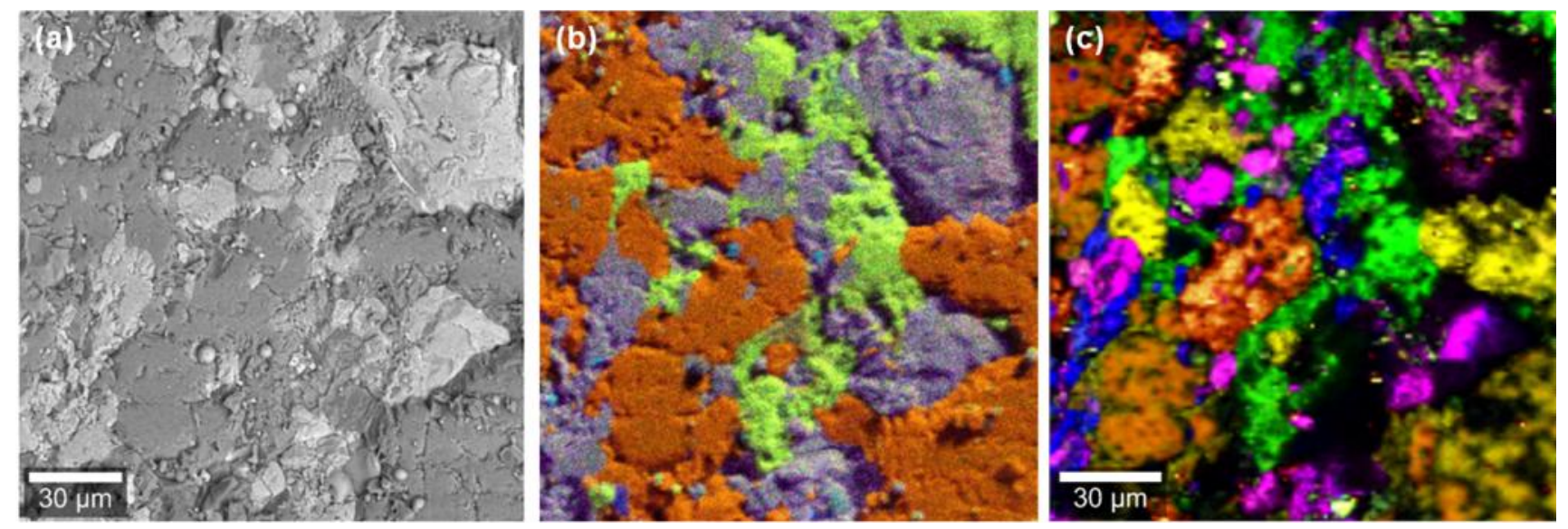

Figure 1. RISE imaging of a mineral phase of a rock section derived from a drill core (KF0066A01, $10.2 \mathrm{~m}$ ) from the Äspö Hard Rock Laboratory. SEM (a), EDX (b) and Raman (c) images acquired from the same sample area. With EDX three element groups could be distinguished ( $\mathrm{Si}, \mathrm{O}$ : orange; $\mathrm{Ca}, \mathrm{Fe}$, $\mathrm{Al}$ : grey-purple; $\mathrm{Na}$; green). Raman single spectra acquired from the three distinct regions show characteristic Raman bands of quartz (orange), epidot (brown) and Plagioclase (green). The Raman image reveals further details related to grain orientation within the quartz and epidot phase.

Sample courtesy Christiane Heim, Geoscience Centre GZG, Dept. Geology, University of Göttingen, Germany.
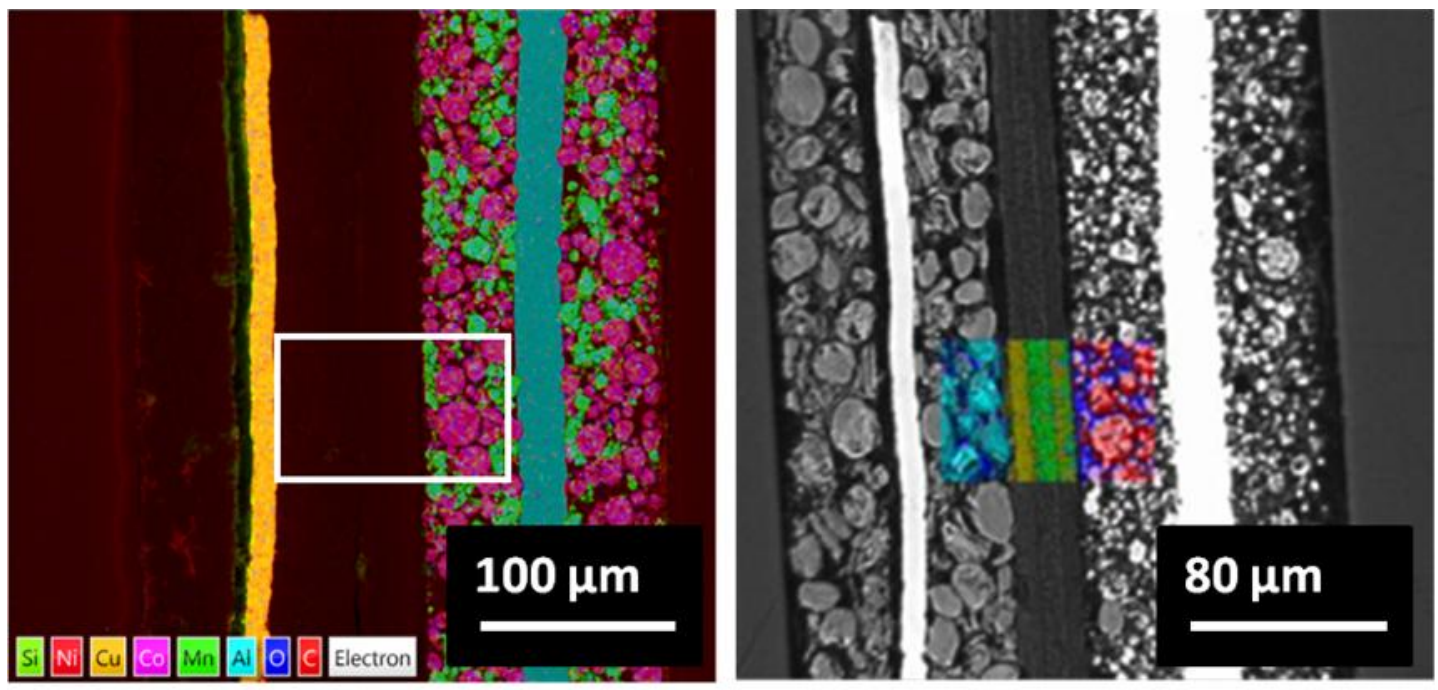

Figure 2. RISE imaging of a Lithium ion Battery: SEM image with EDX mapping (a) and light microscopy with Raman overlay from the same sample area.

Sample courtesy C. Weisenberg, A. Kopp Institut für Materialforschung, Hochschule Aalen, Germany. 